\title{
STUDY OF PHYSIOLOGICAL AND PATHOLOGICAL BREAST CALCIFICATIONS ON MAMMOGRAPHY
}

Sonia Baweja ${ }^{1}$, Saurabh Baweja ${ }^{2}$, Asha Dixit ${ }^{3}$, Abhijeet Yadav ${ }^{4}$, Monika Shrivastava ${ }^{5}$
1. Assistant Professor, De partment of Anatomy, Gandhi Medical College, Bhopal
2. Consultant Radiology, De partment of Radiology, Jawaharlal Nehru Cancer Hospital, Bhopal.
3. Professor \& Head of the Department, Department of Anatomy, Gandhi Medical College, Bhopal
4. PG Student, Department of Anatomy, Gandhi Medical College, Bhopal
5. PG Student, Department of Anatomy, Gandhi Medical College, Bhopal

\section{CORRESPONDING AUTHOR: \\ Sonia Baweja, \\ E 1/137 Arera Colony Bhopal, MP PIN 462016. \\ E-mail:saureja26@yahoo.com}

\section{HOW TO CITE THIS ARTICLE:}

Sonia Baweja, Saurabh Baweja, Asha Dixit, Abhijeet Yadav, Monika Shrivastava. "Study of physiological and pathological breast calcifications on mammography". Journal of Evolution of Medical and Dental Sciences 2013; Vol2, Issue 26, July 1; Page: 4798-4804.

ABSTRACT: INTRODUCTION: Breast calcifications are too small to feel. They can be seen on mammography. They are common and found in about half of all mammograms of females aged 50 and older and one in 10 mammograms of young women. The initial assessment of calcification is made to determine if they confirm well established benign morphologies. Calcifications with benign morphologies require no further investigation. If the characteristics of the calcification are such that they cannot be reliably classified as due to benign processes, then additional evaluation is indicated. MATERIAL AND METHODS: A total of 100 mammograms showing calcification in females of different age group were studied. 4 age groups were included 35-44, 45-54, 55-64 and 65-74yrs.The size, morphology and distribution of calcification was studied.

OBSERVATION AND CONCLUSION: Calcification is a frequent finding in both benign and malignant breast diseases. Distribution of calcification is often a clue to their etiology when no evident mass is present.

KEY WORDS: Mammography, calcification, fibroadenoma, benign.

INTRODUCTION: Breast calcifications are tiny calcium deposits that sometimes form in the breast as a woman ages. Calcifications are too small to feel. They can be seen on mammography where they appear as small bright white spots. Calcifications are common and found in about half of all mammograms of female aged 50 \& older and about 1 in 10 mammogram of young women. The recognition that certain patterns of calcium deposition are associated with malignant processes has made possible the early detection of many cancers. The detection of these clustered microcalcifications associated with cancer is unique to mammography and one of its most important functions.

Calcifications as small as 0.2 and $0.3 \mathrm{~mm}$ are visible on mammography. There are two types of calcification. 
1. Macro calcification - Almost always related to a benign breast condition. They appear large and round on a mammogram \& need no further follow up.

2. Microcalcification - Smaller and more numerous than macro calcification. Radiologist looks at size, shape and pattern of microcalcifications. Follow up mammograms or a biopsy may be needed.

The mammographic appearance of the breast depends on the relative amounts of fat and glandular tissue. The young woman's breast contains a large proportion of glandular tissue which appears as soft tissue density on the mammogram, in older women, when involution of the glandular tissue has occurred, most of the breast tissue appears of fatty density. The glandular and ductal tissue constitutes the breast parenchyma. A patient's future risk of breast cancer can be predicted from the parenchymal pattern seen on her baseline mammographic examination ${ }^{1,2}$. The breast contains 15-20 lobes, each with a duct opening in a nipple. The main duct branches repeatedly within the breast and the most distal branches of the duct system are called the terminal ducts. The terminal duct consists of extra lobular \& intralobular portions. The intralobular portion, together with the acini, forms a lobule. The extra lobular terminal duct and the lobule form a terminal ductal lobular unit. The terminal ductal lobular unit is the site of origin of most malignant and benign diseases of the breast ${ }^{3}$. Most calcifications in the breast form either in terminal ducts (intraductal calcifications) or within the acini (lobular calcification).

Coarse calcification (more than $1 \mathrm{~mm}$ thickness) located within the margin of a mass indicates a calcified fibroadenoma. A thin (1 mm or less) curvilinear calcification at the rim of a mass indicates a benign lesion such as a cyst, fat necrosis or fibroadenoma; most of these, especially long, thin, arc-like calcifications are cysts or fat necrosis. Coarse calcification within the body of a well circumscribed mass usually indicates a fibroadenoma, but may at times be seen in carcinoma ${ }^{4}$.

MATERIAL AND METHODS: The study was done in Jawaharlal Nehru Cancer Institute Bhopal in association with Gandhi Medical College, Bhopal. A total of 100 mammograms with calcifications were studied. Females of age group 35-74 years divided into four groups decade wise were included .All mammograms with calcification were included and without calcification were excluded. Effective mammography requires consistent high quality images with optimum film density and contrast, high resolution, and low radiation dose. The standard examinations for women undergoing either symptomatic mammography or their first screening examination consisted of a lateral oblique and a cranio-caudal view of each breast ${ }^{3}$. Ideal mediolateral oblique positioning should permit the breast to be imaged from high in the axilla down to and including the inframammary fold. The term oblique applies to the plane of breast compression. In craniocaudal projection, compression is usually applied from top of the breast with the detector system under the caudal surface. The gantry is positioned with the beam perpendicular to the floor ${ }^{5}$.The properly positioned mediolateral oblique and craniocaudal view should image as much of the breast as possible.

Mammograms are viewed in optimum lighting conditions with light from around the films and extraneous light in the viewing room excluded as far as possible. The overall parenchymal pattern of the breast is assessed. The mediolateral oblique and craniocaudal views are studied with the right and left breast films 'back to back' so that symmetry of breast tissue can be examined ${ }^{3}$. 
OBSERVATION AND RESULTS: In all 100 mammograms, both craniocaudal and mediolateral oblique views were studied. Analysis was also done with magnifying glass. The following observations (Table no 1) were made:

\begin{tabular}{|l|l|l|l|}
\hline S.No. & $\begin{array}{l}\text { No. of } \\
\text { cases }\end{array}$ & Pattern of calcification & Associated findings \\
\hline 01 & 17 & Microcalcification & $\begin{array}{l}\text { 15 had malignancy(cluster) 2 was benign } \\
\text { with diffuse calcification }\end{array}$ \\
\hline 02 & 32 & $\begin{array}{l}\text { Macro calcification (rounded single } \\
\text { as well as multiple) }\end{array}$ & $\begin{array}{l}\text { 12 had benign breast changes 20 had no } \\
\text { abnormality }\end{array}$ \\
\hline 03 & 20 & Vascular & All cases were above 50 years of age \\
\hline 04 & 7 & Curvilinear/rim & 5 had cysts, 2 had fat necrosis \\
\hline 05 & 12 & Popcorn/coarse & All had involuting fibroadenoma \\
\hline 06 & 3 & Suture & All cases had history of lumpectomy \\
\hline 07 & 7 & Pleomorphic & All cases had malignancy \\
\hline 08 & 2 & Dystrophic irregular & Cases had previous history of radiation \\
\hline
\end{tabular}

TABLE NO 2: TO SHOW PATTERNS IN VARIOUS AGE GROUPS

\begin{tabular}{|l|l|l|l|l|}
\hline Pattern of calcification & $\mathbf{3 5 - 4 4}$ yrs & $\mathbf{4 5 - 5 4}$ yrs & $\mathbf{5 5 - 6 4}$ yrs & $\mathbf{6 5 - 7 4}$ yrs \\
\hline Microcalcification & 2 & 4 & 6 & 5 \\
\hline Macro calcification & 16 & 10 & 4 & 2 \\
\hline Vascular & 0 & 3 & 6 & 11 \\
\hline Popcorn & 3 & 4 & 4 & 1 \\
\hline Pleomorphic & 1 & 1 & 0 & 5 \\
\hline Curvilinear & 2 & 3 & 2 & 0 \\
\hline Suture & 1 & 0 & 2 & 0 \\
\hline Dystrophic & 0 & 0 & 1 & 1 \\
\hline
\end{tabular}

DISCUSSION: The etiology of a mass can frequently be inferred by associated calcification. Calcium deposits are extremely common in the breast. Stomper and associates reported that they found calcification on $8 \%$ of the mammograms performed for women between the ages of $25 \& 29$ with a steady increase to $86 \%$ of the mammograms performed on females between the ages of $76 \& 796$. However the present study included mammograms with calcifications.

Vascular calcifications (fig.1) are linear or form parallel tracks that are clearly associated with blood vessels. They are virtually always related to advanced age ${ }^{7}$. In the present study, the incidence of vascular calcification increased with advancing age and was associated with atherosclerotic changes.

Coarse or popcorn like calcification (Fig. 2) is produced by involuting fibroadenomas. These calcifications usually do not cause a diagnostic problem. Our study correlated well with the study done by Dupont et al which suggested that fibroadenomas are not at risk for malignant change but they are indicator of future overall risk ${ }^{8}$.

Round \& punctate calcifications are $0.5-1 \mathrm{~mm}$ in size and frequently form in the acini of the terminal duct lobular unit. When calcifications are smaller than $0.5 \mathrm{~mm}$ term punctate is used. These can be 


\section{ORIGINAL ARTICLE}

seen in fibrocystic changes or adenosis, skin calcification, skin talc and rarely in Ductal carcinoma in situ.

Eggshell or rim calcifications are very thin benign calcifications that appear as calcium is deposited on the surface of a sphere. Calcification in the wall of cysts and fat necrosis produce these thin deposits.

Milk of calcium is the term associated with the process in which calcium precipitates in benign cysts ${ }^{9}$. The calcification is described as meniscus shaped or tea cup shaped. There is a slight suggestion that milk of calcium may represent a risk factor for breast cancer. Sickles reviewed more than 200 cases of mammographically evident milk of calcium. Eight of 200 cases had associated malignancy but it likely that these women had other reasons for malignancy. In the present study, the egg shell and meniscus shaped calcifications were clearly associated with benign entities.

Suture calcifications (Fig.3) are typically linear or tubular in appearance and represent calcium deposits on suture material before it is reabsorbed ${ }^{10}$. They are common in post irradiated breast and when extensive surgery has been performed.

Dystrophic calcifications are larger than $0.5 \mathrm{~mm}$ and have a lucent center. They are seen in irradiated breast or following trauma. They develop 3-5 years after treatment. It is important to differentiate them from a recurrent malignancy.

Calcifications with high probability of malignancy are fine pleomorphic and fine linear or fine linear branching. Calcifications associated with cancer have been variously described as having comma, shapes with pointed projections and irregular surfaces. Egan and associates found that among 115 patients biopsied for microcalcifications alone, all lesions diagnosed as cancer had at least five visible flecks of calcium in a $0.5 \times 0.5 \mathrm{~cm}$ field. The probability of malignancy was zero when there were fewer than five calcific particles.

Finally, distribution of calcification is often a clue to their etiology. Diffuse or scattered distribution (Fig. 4) is typically seen in benign entities. Even when clusters of calcifications are scattered throughout the breast, this favors a benign entity.

Regional distribution favors a non-ductal benign distribution. Segmental distribution favors a ductal distribution. Virtually all calcifications that form in breast cancers form in the intraductal portion of cancer, although multiplying cells can expand the duct. This occurs when tumour diameter enlarges beyond $180 \mu \mathrm{m}^{11}$.

Clustered calcifications are seen both in benign and malignant diseases. If clusters are scattered throughout the breast, this is suggestive of benign entity while a single cluster of calcification favors a malignant entity. One of the major challenges of mammography is distinguishing punctate calcification of benign adenosis from the clustered microcalcification of malignancy. Adenosis calcifications occur in the terminal acini and are round, scattered and usually bilateral. However there is much overlap between the appearances of benign and malignant calcifications ${ }^{12}$.

Calcification is a frequent finding in both benign and malignant breast diseases. Where no mass is present, they may represent the only evidence of such disease. Certain aspects of their appearance should suggest the possibility of malignancy ${ }^{13-16 .}$

CONCLUSION: The likelihood of carcinoma is proportional to the concentration of calcific particles. Therefore, one should consider biopsy of any focus of three or more microcalcifications if no other 
calcifications are present elsewhere in the breast or in the opposite breast. Benign and malignant processes may produce similar patterns of calcium deposition. Analysis of location, size, morphology, number, distribution and any associated findings of the deposit can however eliminate many from suspicion.

\section{REFERENCES:}

1. Kalisher L, Mc. Lelland R, Feig SA 1983 Mammographic patterns and breast cancer risk In: Feig SA, Mc. Lelland R (eds) Breast Carcinoma: Current diagnosis and treatment. Masson, New York PP 77-88.

2. Wolfe JN, Alber S, Belle S, Salane M 1982 Breast Parenchymal Patterns: analysis of 332 incident breast carcinomas. Am J Roentgenol 138: 113-118.

3. M.J. Michell. Textbook of Radiology and Imaging. $6^{\text {th }}$ edn. Volume 2. London: Churchill Livinstone, 1998. Chapter 53: 1429-50.

4. Feig SA, Piccoli CW. Grainger and Allison's diagnostic radiology. $3^{\text {rd }}$ edn. vol. 3. New York: Churchill Livingstone, 1997. Chapter 92A: 1995-2015.

5. Kopans. Breast Imaging. 2nd edn. Philadelphia: Lippincott, 1998. Chapter 10: 171-208.

6. Stomper PC, D;Souza DJ, DiNitto PA, Arredondo MA. Analysis of parenchymal density on mammogram in 1353 women 25-79 years old Am J Roentgenol 1996; 167: 1261-1265.

7. Kopans. Breast Imaging. $2^{\text {nd }}$ edn. Philadelphia: Lippincott, 1998. Chapter 13: 315-339.

8. Dupont WD, Page DL, Pari FF, et al. Long term risk of breast cancer in women with fibroadenoma. N Engl J Med 1994; 331 : 10-15.

9. Sickles EA, Abele JS. Milk of calcium within tiny benign breast cysts. Radiology 1981; 141 : 655-658.

10. Stacey - Clear A, Mc Carthy KA, Hall DA, et al. Calcified suture material in he breast after radiation therapy. Radiology 1992; 183: 207-208.

11. Mayr NA, Staples JJ, Robinson RA, et al. Morphometric studies in ntraductal breast carcinoma using computerized image analysis. Cancer 1991; 67:2805-2812.

12. Sickles EA 1984 Mammographic features of early breast cancer. AJR 143: 461-464.

13. Millis RR Davis R, Stacey AJ 1962. The detection and significance of calcifications in the breast: a radiological and pathological study. Br J Radiol 49: 12-26

14. Murphy WA, De Schryver- Kecskemati K 1978 Isolated clustered microcalcification in the breast: radiologic - pathologic correlation Radiology 127: 335-341.

15. Sigfusson BF, Anderson I, Aspegren K, Janzon L, Linell F, Ljungberg 01983 Clustered breast calcifications. Acta Radiol Diagn 24: 273-281.

16. Wolfe JN 1977 Xeroradiography Breast Calcifications. Thomas, Springfield. 


\section{ORIGINAL ARTICLE}

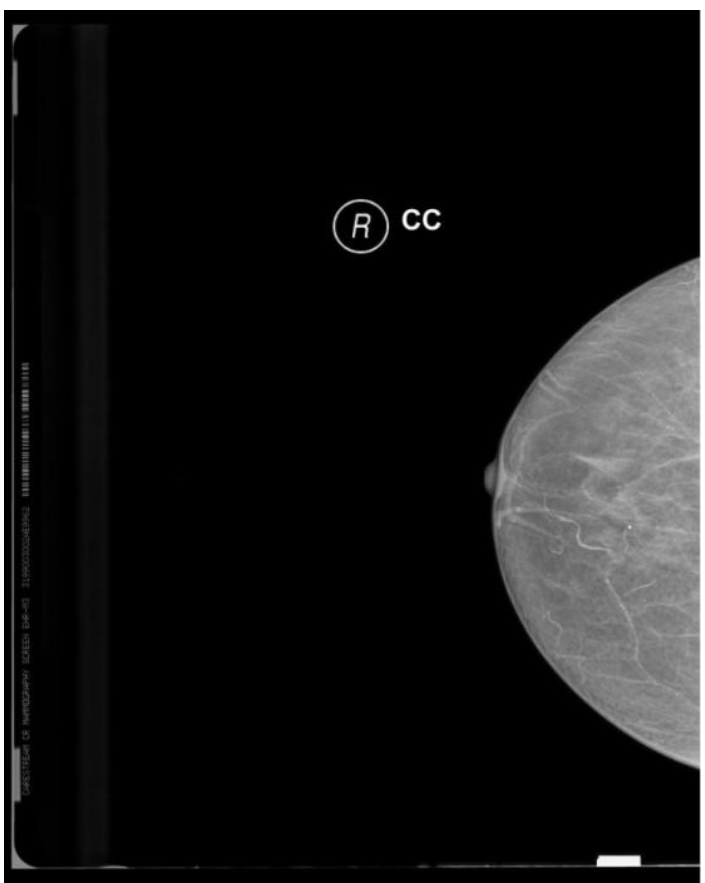

FIG. 1: CC view showing parallel vascular calcification (physiological)

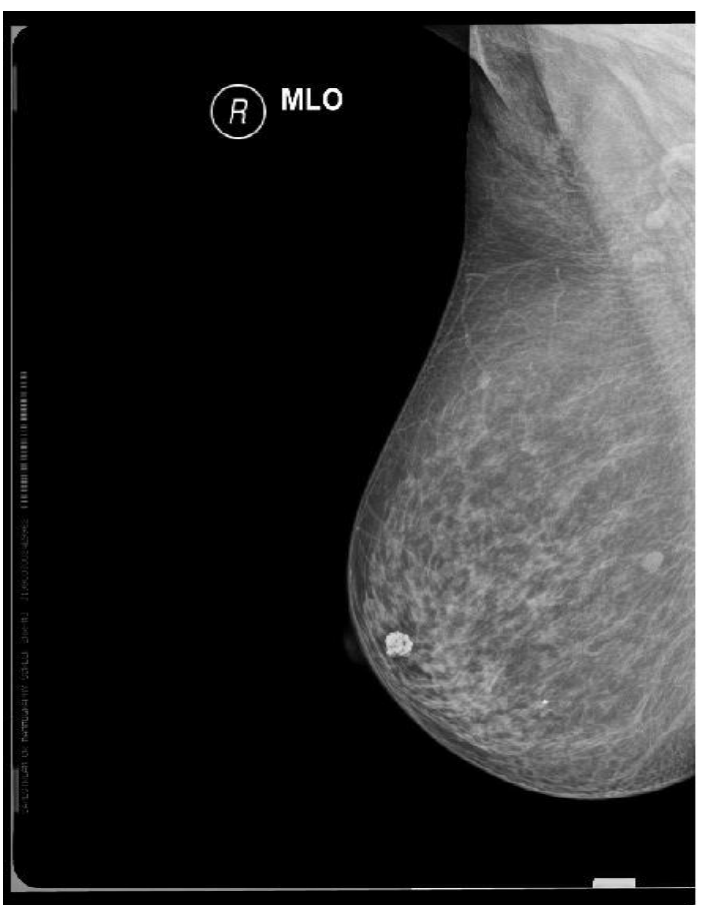

Fig. 2: MLO view showing popcorn calcification suggestive of involuting fibroadenoma 


\section{ORIGINAL ARTICLE}

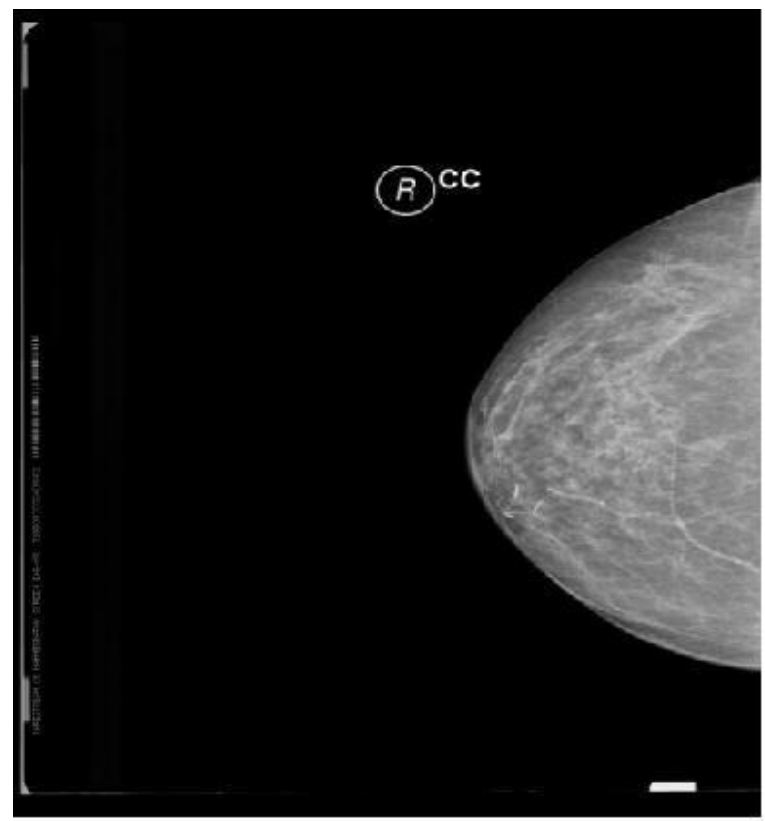

Fig. 3: CC view showing suture calcification.

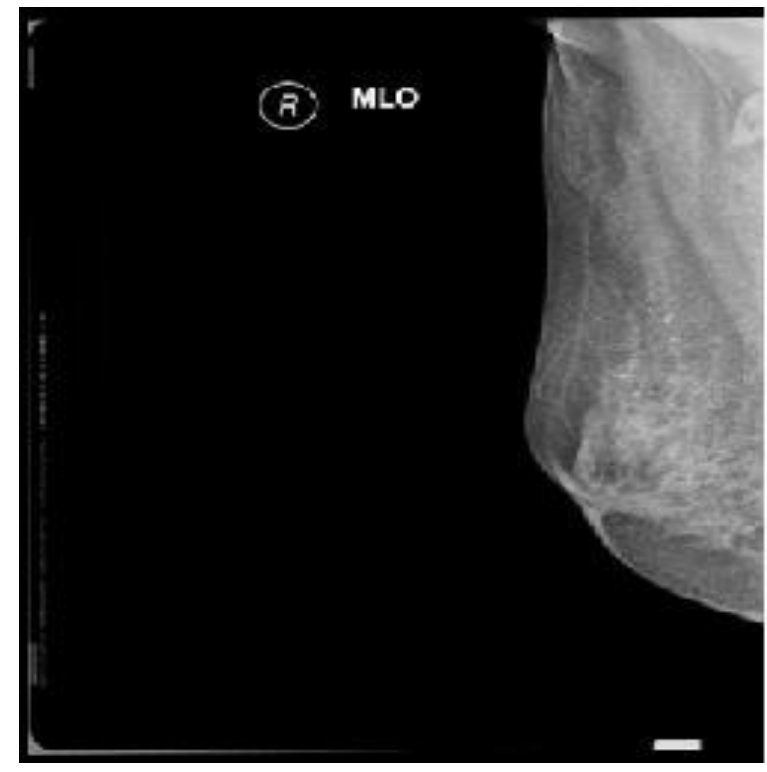

Fig. 4: MLO view showing scattered macro calcification suggestive of benign nature 